\title{
The road to extinction: commons with capital markets ${ }^{1}$
}

\author{
Jayasri Dutta $^{2} \quad$ Colin Rowat $^{3}$
}

This version: January 22, 2004

\footnotetext{
${ }^{1}$ We wish to thank Bob Anderson, Ralph Bailey, Herakles Polemarchakis, Peter Postl and seminar participants at the University of Birmingham and the Midlands Game Theory conference.

${ }^{2}$ Department of Economics, University of Birmingham; j.dutta@bham.ac.uk

${ }^{3}$ Department of Economics, University of Birmingham; c.rowat@bham.ac.uk
} 


\begin{abstract}
Competitive agents extract in continuous time from a commons. Capital market access allows them to both save and borrow against their extraction stream. When the commons asset grows more quickly than the privately stored one, multiple equilibria are found for intermediate commons endowments. One of these has the extinction date and welfare decrease in the endowment, a resource curse. When the commons asset grows less quickly than the privately stored one, there is a unique extinction date for each endowment level. In the limit, as marginal extraction costs become constant, 'jump extinctions' occur. In cases with multiple equilibria: welfare is increased for low initial stock levels when agents do not have access to capital markets, but decreased otherwise; and an extraction tax reduces welfare in the 'cursed' equilibrium, increases it in the other finite extinction equilibrium and expands the set of commons stocks that are never extinguished.
\end{abstract}

Key words: commons, storage, capital markets, rational expectations equilibrium, extinction, resource curse, multiple equilibrium

JEL classification numbers: C73, D91, O17, Q21 


\section{Introduction}

In almost any contemporary common access problem, those drawing on the resource also have access to capital markets. Thus, the proceeds of their extraction need not be immediately consumed; they may be saved, or even borrowed against. In spite of this, relatively little attention has been paid to the effects of capital market access on commons problems. This paper and a companion (Dutta and Rowat, 2003) seek to redress this. A particular interest of the present paper is effect of access to capital markets on the 'extinction' of the commons.

The simplest commons analyses, dating to Gordon (1954), are static: free entry allows the rents associated with a natural resource to be competed away - the 'tragedy of the commons'. The free entry assumption motivates competitive rather than strategic solution concepts. The static framework obviates questions of capital market access.

This is, however, a large dynamic commons literature which concerns itself with intertemporal issues. To date, the bulk of this has not allowed capital market access, forcing each agent into intertemporal autarky. See Mirman (1979); Levhari and Mirman (1980); Benhabib and Radner (1992); Dutta and Sundaram (1993); Dockner and Sorger (1996); Sorger (1998) for examples of this literature. ${ }^{1}$ Thus, subject to Inada conditions, it is never in anybody's interest to ransack to the point of extinction. Of course, the commons are often overfished or overgrazed relative to what a benevolent planner would ordain. ${ }^{2}$

More recently, the possibility of relating consumption and extraction through a intertemporal constraint, rather than constraints in each period, has been explored. As we outline, this has been done in a number of ways, from simple storage technologies to access to capital markets. The present paper focusses on this latter possibility. We argue that the possibility of private storage can remove Inada's prohibition, thereby allowing extinction, and possibly even greater inefficiency.

One of the earliest examples of this literature is Sinn (1984). In this,

\footnotetext{
${ }^{1}$ Houba, Sneek, and Várdy (2000) present an interesting twist on this literature in their alternating offers model played concurrently with a fishing game to determine the size of the pie.

${ }^{2}$ Exceptions to this result exist. Dutta and Sundaram (1993) present a discrete time example in which under-exploitation of the commons can occur when trigger strategies are defined on the state variable; they note that this idea is also found in Fudenberg and Tirole (1983). Their equilibria remain inefficient. In continuous time, Benhabib and Radner (1992) find ranges of initial conditions that allow trigger strategies yielding efficient equilibria. Dockner and Sorger (1996) and Sorger (1998) also derive conditions under which equilibria are efficient.
} 
oligopolistic firms extract oil from underground reserves, either to sell immediately or store above ground. As firms own private oil fields, which only interact through seepage, is a problem of oligopolistic competition in the product market rather than extraction from a commons.

Kremer and Morcom (2000) consider a model with genuine storage. Competitive poachers may kill elephants (an open access resource) and store their ivory tusks at the opportunity cost. In contrast to the preceding literature that they cite, they argue that there may be

multiple equilibria for open-access renewable resources used in the production of storable goods, because if others poach, the animal will become scarce, and this will increase the price of the good, making poaching more attractive.

In our model, there is an interval of initial commons stock levels within which multiple equilibria are found. In particular, there are three, two involving extinction in finite time; extinction does not occur in the remaining one.

Homans and Wilen (2001) also explore competitive equilibria. A fixed cost of fishing restricts the number of agents to be finite. Further, access to the commons is regulated, a framework that they regard as more consistent with existing fisheries than open-access. Their attention is focussed on the market for caught fish: fish caught during the fishing season must satisfy a year's consumer demand; that sold immediately is sold as fresh, and therefore at a higher price than that sold after being frozen. They argue that increased rents in the fishing industry both induce entry and shorten the fishing season, thus causing more fish to be sold on the inferior market. ${ }^{3}$

The most recent paper in this literature is Gaudet, Moreaux, and Salant (2002), which also considers competitive equilibria in a commons environment with private storage. ${ }^{4}$ Its motivating examples are powerful: very rapid depletion of underground oil reserves, annual fishing quotas and groundwater. As average extraction costs become constant, their model captures these 'jump extinctions', which are similar to speculative attacks. We also obtain this result as a limit case of our model: generically, the road to extinction is smooth in our model.

Two earlier, related papers address considered the possibility not of costly storage, but of full access to capital markets: yield-bearing storage and costly borrowing. Tornell and Velasco (1992) ask why capital flows from poor countries to rich countries. They interpret poor countries as having not just high

\footnotetext{
${ }^{3}$ Fish raised in farms are often fed fish pellets (Weiss, 2002). Such farms may therefore be a way of converting cheaper (frozen) fish into more expensive fresh fish.

${ }^{4}$ Their suggestion that the earlier literature neglects storage uses an unfortunate example given that cows are ruminants.
} 
rates of return, but weak property rights so that domestic returns can be appropriated by anyone with equal facility; thus, domestic investment is investment in a commons. Capital flight corresponds to private storage of pillage from common property in a wealthy country, thus at lower rates of return. With perfect international capital markets, borrowing and lending may occur at the same rate.

As there is a small number of interest groups, their solution concept is a stationary Markov perfect equilibrium.

A sequel, Tornell and Lane (1999), again imagines a country with weak institutions. Now, the interest groups compete for control over government revenues, raised by taxing the formal sector. Thus, the formal (high return) sector becomes a commons; the informal (low return) sector is the medium for private storage.

These papers suggest a very different interpretation of the commons problem than the standard one, in which the tragedy is that of the rents' dissipation. In their view, the commons is associated with poorly defined property rights, weak institutions, and poverty. ${ }^{5}$ From this point of view, the tragedy of these commons may be their persistence, not their extinction. By contrast, richer countries have likely enclosed their commons at some point in the past.

Thus, to the extent that extinction corresponds to enhanced property rights, it may be a desirable outcome. The standard interpretation of the tragedy reminds us, though, that this is not assured: the costs of enforcing property rights may dissipate rents. ${ }^{6}$

Equilibrium extinction may therefore be seen as voluntary privatization: a self-enforcing move from common to private property. Enclosures, gold rushes or, for that matter, the acquisition of Mesopotamian antiquities by private collections are all pertinent examples: are such equilibrium allocations of property rights likely to lead to efficiency? ${ }^{7}$ If so, might this provide a mechanism for a 'resource curse', whereby societies better endowed with resources may take longer to move to private property? (See Sala-i-Martin and Subramanian (2003) for a review of this literature.)

A weakness of Tornell and Velasco (1992) and Tornell and Lane (1999) in this context is that their models rule out the possibility of full extinction or enclosure. As extraction is costless in their model, an extreme version of the limit case of Gaudet et al. (2002), one might expect this to occur instantly.

\footnotetext{
${ }^{5}$ In the popular debate, this view has been argued forcefully by de Soto (2000).

${ }^{6} \mathrm{~A}$ Washington lawyer advising on investment in Iraq "believes security costs could in some cases total $25 \%$ of a contract's value" (Richter, 2003).

${ }^{7}$ In a twist on the usual story, dogs, which allowed themselves to be enclosed by us, are abundant while wolves, which did not, are not.
} 
Even more counter-intuitively, Tornell and Velasco (1992) claim that the possibility of foreign storage of pillage

puts a floor on the common-access asset's rate of return and, thus, a ceiling on the appropriation rate. If this constraint is binding, interest groups will be forced to reduce their appropriation rate. This will increase aggregate capital accumulation, ameliorate the tragedy of the commons, and increase welfare.

To explore these questions further, this paper looks at perhaps the simplest possible representation of the problem, close to the industry standard on tragedy of the commons, but with capital market access. A model is presented in Section 2. This closely follows the structure of the models presented in Tornell and Velasco (1992) and Tornell and Lane (1999), generalising in two ways. First, extraction may be costly. Second, strategies are not required to be shares of existing stocks. Without this latter generalisation, extinction is not technically possible.

While our model is consistent with the interpretations given to high and low rates of return in both Tornell and Velasco (1992) and Tornell and Lane (1999), it is also consistent with a third variant: there is only one rate of return, but the costs of enclosing resources to protect them against expropriation reduce the net rate of return on privately held resources to the lower rate. Thus, the difference between the high and low rates may be seen as reflecting the weakness of property rights. This interpretation is very general, encompassing situations of both economic and biological growth.

The examples motivating Tornell and Velasco (1992) and Tornell and Lane (1999) have an illicit flavour to them. While the ability to bank one's 'plunder' seems relatively uncontentious, the possibility of borrowing against future plunder does seem less plausible. The empirical support for this practice does seem specialised: Ross (2003, pp. 32-) discusses 'booty futures' in the context of civil wars.

This illicit flavour is unnecessary: a refinery may borrow against its expected profits, calculated on the assumption that it will be able to discharge combustion byproducts into the communal atmosphere.

Following Gaudet et al. (2002), Kremer and Morcom (2000) and Homans and Wilen (2001), the solution concept is a rational expectations equilibrium: there are no barriers to entry; individuals are small and do not take account of the impact of their actions on the evolution of aggregate capital stock. This is introduced in Section 3. (In the companion paper, Dutta and Rowat (2003), we evaluate the extent to which strategic, subgame perfect equilibria inherit these extinction properties. We find that they do, even for very small numbers of agents.) 
As noted, we find the Gaudet et al. (2002) 'jump extinction' as a special case. More generally, low initial commons stocks correspond to unique solutions, with extinction in finite time; an intermediate range of initial commons stock produces the multiple equilibria already mentioned; initial stocks above this level are never exhausted.

Section 4 compares competitive outcomes with capital market access to autarkic ones in which competitive agents do not have access to capital markets. In the example analysed, welfare under autarky is higher for low initial stock levels. Once commons stocks are sufficient to support multiple extinction dates, superior welfare can be obtained with capital market access.

Section 5 considers the consequences of an extraction tax; it has the effect of shrinking the interval of commons stock levels that lead to multiple extinction dates. This shrinks the domain over which the 'cursed' equilibrium can arise and expands the set of commons stocks that are never extinguished. Its effect may be reversed, however, when the government imposing the tax is strong.

Section 6 concludes.

\section{The model}

Time, indexed by $t$, passes continuously toward an infinite horizon. At every point in time, a continuum of individuals, indexed by $i$ and distributed on the unit interval with cumulative distribution $F$, decides on extraction, $x_{i}=$ $\left\{x_{i}(t)\right\}$, and consumption, $c_{i}=\left\{c_{i}(t)\right\}$.

There is a single consumption good, whose stock, $k=\{k(t)\}$, is common property. It grows at rate $a$. At each point in time, individuals extract, in total, $x(t)=\int_{i} x_{i}(t) d F(i)$ from the commons, storing it as their private property. ${ }^{8}$ Thus, $x_{i}(t)$ is the (finite) extraction rate at time $t$ of infinitesimal agents $d F(i)$.

The extraction path, $x=\{x(t)\}$, defines the initial value problem

$$
\dot{k}(t)=a k(t)-x(t), k(0)>0
$$

whose solution is the path of the capital stock whenever $k(t)>0$.

An extinction date is the earliest $T \geq 0$, such that $k(T)=0$. If $\lim _{t \rightarrow \infty} k(t)$ $>0$, then $T=\infty$, which corresponds to non-extinction. Since $k(T)=0 \Rightarrow$ $x(T)=0$, the capital stock is absorbed at 0 and

$$
k(t)=\max \left\{0, k(0) e^{a t}-\int_{0}^{t} e^{a(t-\tau)} x(\tau) d \tau\right\} .
$$

\footnotetext{
${ }^{8}$ This general specification is not generally taken advantage of. We often replace $d F(i)$ with the uniform $d i$.
} 
Equation 2 describes the unique solution to initial value problem whenever $x(t)$ is continuous over $[0, T)$ (Walter, 1998, p.28). Thus, aggregate extraction is admissible if $x(t)$ is continuous over $[0, T) .{ }^{9}$

It may be seen immediately that restricting extraction rates to be shares of the commons stock, as in Tornell and Velasco (1992), precludes extinction. Let $x_{i}(t)=\sigma_{i} k(t)$ and $x(t)=\sigma k(t)$. Then the solution to equation of motion 1 is

$$
k(t)=\max \left\{0, k(0) e^{(a-\sigma) t}\right\} .
$$

Thus, the capital stock either grows to infinity or asymptotes to zero. This argument applies regardless of whether the $\sigma_{i}$ are derived competitively or strategically.

Goods extracted from the commons are presented to capital markets. If saved, they earn a return of $r \leq a$. Equally, future extraction may be borrowed against, smoothing consumption, at the same rate.

Capital market access enables a decomposition of individuals' optimization problems: instantaneous feasibility constraints on consumption are replaced by a single intertemporal budget constraint:

$$
\int_{0}^{\infty} e^{-r t}\left(c_{i}(t)-x_{i}(t)\right) d t \leq 0 .
$$

Thus, $x_{i}(t)<c_{i}(t)$ implies borrowing against future extraction. The possibility of default is not considered.

It may happen, for $r$ high enough, that individuals' chosen paths satisfy $x_{i}(t)>c_{i}(t)$ up to some $T$. We do not impose this as a constraint on individuals.

Individuals choose extraction and consumption paths to maximize utility subject to their budget constraint, 3 , and to a feasibility (or non-negativity) constraint

$$
k(t)=0 \Rightarrow x_{i}(t)=0 .
$$

Their utilities are

$$
V_{i}\left(c_{i}, x_{i}\right)=\int_{0}^{\infty} e^{-\rho t}\left(U\left(c_{i}(t)\right)-\frac{C\left(x_{i}(t)\right)}{\theta_{i}}\right) d t
$$

where utility and cost functions are

$$
U(c)=\frac{c^{1-\alpha}}{1-\alpha}, \quad \alpha \geq 0 \quad \text { and } \quad C(x)=\frac{x^{1+\gamma}}{1+\gamma}, \quad \gamma \geq 0 .
$$

\footnotetext{
${ }^{9}$ Dockner, Jørgenson, Long, and Sorger (2000, p. 40, Definition 3.1) use 'feasible' in place of 'admissible'.
} 
Utility from consumption is concave while extraction costs are convex: $U^{\prime \prime} \leq$ $0, C^{\prime \prime} \geq 0$. Values of $\alpha>1$ yield utility function more concave than the log, which corresponds to $\alpha=1$.

It may seem natural to set $\gamma=0$, linear extraction costs. This may be interpreted as a situation in which there is a competitive market for the inputs into a CRS extraction function. As we shall see, aggregate extraction grows exponentially over time. Thus, insofar as this interpretation implies a labour market, the wages of a fixed supply of labourers may be bid up. This is consistent with $\gamma>0$.

It will be seen that $\gamma=0$ produces bang-bang style solutions. Thus, we treat this case separately from the generic case, in which we assume $\gamma>0$. Similarly, although techniques can be developed to handle $\alpha=0$, we work with $\alpha>0$.

To ensure finite valuations, we impose Uzawa-integrability conditions:

$$
(1-\alpha) r<\rho<(1+\gamma) r
$$

The origins of these will become clearer later.

Individuals differ in their costs of extraction or access to common property. Thus, $\theta_{i}>\theta_{j}$ implies $i$ has easier access. Alternatively, individuals may also be regarded as being indexed by $\theta_{i}>0$.

A rational expectations equilibrium is described by sequences $\left\{k \geq 0, c_{i} \geq 0, x_{i} \geq 0\right\}$ and an extinction time, $T$, such that $k(t)=0$ for all $t \geq T$, where

1. individuals choose consumption and extraction paths to maximize utilities subject to their budget and feasibility constraints;

2. the evolution of the capital stock is determined by the aggregate extraction path.

The equilibrium is competitive in the standard sense: although individuals' aggregate behaviour influences the economic environment, $T$ in this case, they disregard their individual effects on it. Thus, our agents are extinction date takers.

In the following, we solve the model for equilibrium, and, in particular, for extinction dates.

\section{Rational equilibria}

The assumption that individuals can borrow and lend at rate $r$ allows the decomposition of their problem of determining extraction and consumption into two separate problems: 
1. the consumption-smoothing problem: choose $c_{i}(t)$ given total wealth $X_{i}(r)=\int_{0}^{\infty} e^{-r t} x_{i}(t) d t$ subject to constraint 3; and

2. the effort-smoothing problem: choose $x_{i}(t)$, given the extinction date $T$ and feasibility constraint, 4 .

\subsection{The consumption smoothing problem}

Agents are viewed as first solving the consumption problem:

$$
\max _{c_{i}(t)} \int_{0}^{\infty} e^{-\rho t} U\left(c_{i}(t)\right) d t \text { subject to equation } 3 .
$$

Defining

$$
X_{i}(r) \equiv \int_{0}^{\infty} e^{-r t} x_{i}(t) d t
$$

facilitates writing the Lagrangian:

$$
\mathcal{L} \equiv \int_{0}^{\infty} e^{-\rho t} \frac{c_{i}(t)^{1-\alpha}}{1-\alpha} d t-\lambda\left[\int_{0}^{\infty} e^{-r t} c_{i}(t) d t-X_{i}(r)\right]
$$

The ensuing Euler equation is standard:

$$
c_{i}(t)=\frac{\rho-r(1-\alpha)}{\alpha} X_{i}(r) e^{\frac{r-\rho}{\alpha} t} .
$$

This is independent of $a, \gamma$ and $\theta_{i}$. Its constant term is determined by the constraint. Substitution into the objective function of equation 6 therefore produces the maximized present value of utility from consumption

$$
U_{B}\left(X_{i}(r)\right) \equiv \nu \frac{\left(X_{i}(r)\right)^{1-\alpha}}{1-\alpha}
$$

where

$$
\nu \equiv\left(\frac{\alpha}{\rho-(1-\alpha) r}\right)^{\alpha}=\frac{1}{\left(r-g_{c}\right)^{\alpha}} .
$$

The Uzawa finite valuation condition for consumption is $\rho>r(1-\alpha)$. This is trivially satisfied if $\alpha \geq 1(U(c)$ is more concave than $\log (c))$. We note that

$$
c_{i}(t)=c_{i}(0) e^{g_{c} t}
$$

where

$$
g_{c} \equiv \frac{r-\rho}{\alpha}
$$

is the growth rate of consumption and $c_{i}(0)$ is chosen to satisfy (3):

$$
c_{i}(0)=\left(r-g_{c}\right) X_{i}(r) .
$$

The Uzawa condition may therefore be expressed as $g_{c}<r$ : consumption growth is lower than the interest rate. 


\subsection{The effort smoothing problem}

The agent's problem is now to

$$
\max _{x_{i}(t) \geq 0} U_{B}\left(\int_{0}^{\infty} e^{-r t} x_{i}(t) d t\right)-\frac{1}{\theta_{i}} \int_{0}^{\infty} e^{-\rho t} C\left(x_{i}(t)\right) d t ;
$$

subject to feasibility constraint 4 .

When $t<T$, the ensuing Euler equation yields

$$
\begin{aligned}
x_{i}(t) & =e^{g t} \frac{\left[\theta_{i} \nu\right]^{\frac{1}{\gamma}}}{X_{i}(r)^{\frac{\alpha}{\gamma}}} \\
& =e^{g t} \frac{\left[\theta_{i} \nu\right]^{\frac{1}{\gamma}}}{\left[\int_{0}^{T} e^{-r t} x_{i}(t) d t\right]^{\frac{\alpha}{\gamma}}} \\
& =e^{g t} \kappa ;
\end{aligned}
$$

where $g=\frac{\rho-r}{\gamma}$ and $\kappa$ is a positive constant. Evaluating this at $t=0$ produces $x_{i}(0)=\kappa$ so that

$$
x_{i}(t)=\left\{\begin{array}{lll}
x_{i}(0) e^{g t} & \text { for } \quad t<T \\
0 & \text { for } \quad t \geq T
\end{array}\right\} .
$$

This allows the term in $x_{i}(t)$ to be removed from the integral for

$$
x_{i}(0)^{\alpha+\gamma}=\frac{\theta_{i}}{\left(\int_{0}^{T} \exp (-(r-g) t) d t\right)^{\alpha}} \nu .
$$

Thus, extraction is smooth until the extinction date, $T$. More significantly, the problem of choosing an extraction path is reduced to a choice of $x_{i}(0)$. Note also that the extraction plan is a function of $\alpha$ : thus, full Fisher separation of extraction (production) and consumptions plans does not occur. This is a consequence of $\gamma>0$ : extraction costs are borne as non-transferable disutility.

The Uzawa condition for extraction is now $g<r$. For now, we assume that $a>r$ as these situations might be expected to produce the most interesting economic behaviour. (When $a \leq r$ maintaining the commons offers no benefits.) Thus, for expositional purposes, we concentrate on $a>g$ at present. Theorem 2 treats the complementary cases as well.

Notice that $g=-\frac{\alpha}{\gamma} g_{c}$; thus, for $r>\rho$, individuals extract early but consume late. The $r=\rho$ case yields $g=g_{c}=0$. This is a potentially important special case (and 'interest rate equals subjective rate of discount' is well justified along equilibrium growth paths). 
Notice also that an expression for extraction as a function of capital stock may now be written. By equations 2 and 11,

$$
k(t)=e^{a t}\left[k(0)-\frac{x(0)}{g-a}\left(e^{(g-a) t}-1\right)\right] .
$$

Thus, when $g \neq a$, extraction cannot be expressed as a linear function of capital stock. ${ }^{10}$

\subsection{Characterising equilibrium}

Define the function

$$
Q_{n}(T)=\int_{0}^{T} \exp (-n \tau) d \tau=\frac{1-\exp (-n T)}{n}
$$

for $n \neq 0$ and $T \geq 0 .{ }^{11}$ Notice that $Q_{n}(T)$ increases with $T$ and decreases with $n$. This allows simplification of equation 12 to

$$
x_{i}(0)=\frac{\left(\theta_{i} \nu\right)^{\frac{1}{\alpha+\gamma}}}{\left(Q_{r-g}(T)\right)^{\frac{\alpha}{\alpha+\gamma}}} .
$$

Integrating over agents then produces the first fundamental equation: the effect of anticipated extinction on extraction:

$$
x(0)=\frac{\mu}{\left(Q_{r-g}(T)\right)^{\frac{\alpha}{\alpha+\gamma}}} ;
$$

where $\mu \equiv \nu^{\frac{1}{\alpha+\gamma}} \int \theta_{i}^{\frac{1}{\alpha+\gamma}} d F(i)$. This equation gives us a map $\mathbf{A}: T \rightarrow x(0)$, monotone decreasing.

We know that $x(0)$ determines the entire path of extractions. We now obtain the second fundamental equation: the impact of extraction on the possible extinction date of common property. As extinction occurs at the lowest $T$ such that $k(T)=0$, it represents a zero of equation 2 :

$$
k(0) e^{a T}=\int_{0}^{T} e^{a(T-\tau)} x(\tau) d \tau .
$$

Thus, by equation 11 and integration over agents,

$$
k(0)=x(0) \int_{0}^{T} e^{-(a-g) \tau} d \tau .
$$

\footnotetext{
${ }^{10}$ In Tornell and Velasco (1992), extraction is assumed to be a linear function of capital stock; their equilibrium concept is different than that here.

${ }^{11}$ When $n=0, Q_{n}(T)=T$.
} 
The Uzawa extraction condition ensures that $r>g$; thus $a>g$ follows. Therefore

$$
\frac{k(0)}{x(0)}=\frac{1-e^{-(a-g) T}}{a-g}=Q_{a-g}(T) \leq \frac{1}{a-g} .
$$

This expression reaches its upper bound as $T \rightarrow \infty$. Therefore $T$ implicitly solves,

$$
Q_{a-g}(T)=\min \left\{\frac{k(0)}{x(0)}, \frac{1}{a-g}\right\}
$$

This equation gives us a map I : $x(0) \rightarrow T$, also monotone non-increasing. Here a low level of initial $x(0)$ guarantees the perpetuation of common property but a level higher than $(a-g) k(0)$ results in extinction in finite time.

The discussion above tells us the likely source of multiple equilibrium in extinction times. Both maps are decreasing: individuals choose to extract more if they believe that the commons will disappear soon; and higher extraction rates speed up extinction.

To formalise the preceding discussion, define

$$
\Psi(t) \equiv \frac{Q_{a-g}(t)}{\left[Q_{r-g}(t)\right]^{\alpha+\gamma}} ;
$$

and $\psi_{*} \equiv \lim _{t \rightarrow \infty} \Psi(t), \psi^{*} \equiv \max _{t} \Psi(t)$.

Theorem 1. There exist $0<k_{L} \leq k_{H} \leq \infty$ such that the following statements are true. Given intervals $I_{L}=\left[0, k_{L}\right), I_{M}=\left(k_{L}, k_{H}\right)$, and $I_{H}=$ $\left(k_{H}, \infty\right)$ :

1. $k(0) \in I_{L}$ implies unique equilibrium with finite extinction;

2. $k(0) \in I_{M}$ implies multiple equilibria, one with non-extinction and two with finite extinction;

3. $k(0) \in I_{H}$ implies unique equilibrium without extinction;

4. $k(0)=k_{L}=k_{H}$ implies a unique equilibrium with non-extinction;

5. $k(0)=$ either $k_{L}$ or $k_{H}$, distinct, implies a unique equilibrium with extinction, and a unique equilibrium with non-extinction.

The following lemmata are used to prove the theorem:

Lemma 1. $\lim _{t \rightarrow 0} \Psi(t)=0$. 
Proof. As $Q_{n}(0)=0$, assessing $\Psi(0)$ requires use of l'Hôpital's rule: differentiating the numerator produces 1 , while doing so to the denominator produces

$$
\frac{\alpha}{\alpha+\gamma}\left[Q_{r-g}(0)\right]^{-\frac{\gamma}{\alpha+\gamma}} e^{-(r-g) 0}=\frac{1}{0} .
$$

Lemma 2. When the Uzawa extraction condition holds and $a>g, 0<\psi_{*}<$ $\infty$.

Proof. By definition, $\lim _{t \rightarrow \infty} Q_{n}(t)=\frac{1}{n}$ when $n>0 .{ }^{12}$ Under the conditions of the lemma,

$$
\psi_{*}=\frac{(r-g)^{\frac{\alpha}{\alpha+\gamma}}}{a-g} .
$$

The Uzawa extraction condition ensures that the numerator is strictly positive. When $a>g$, the denominator is as well, ensuring the results.

Lemma 3. An equilibrium with finite extinction time $T$ satisfies $\Psi(T)=$ $\frac{k(0)}{\mu}$.

Proof. Equations A and I, with $Q_{a-g}(T)=\frac{k(0)}{x(0)}$, are satisfied in equilibrium. The result follows by definition 15 .

Lemma 4. An equilibrium with non-extinction exists iff

$$
\psi_{*} \leq \frac{k(0)}{\mu} .
$$

Proof. Assume that $\psi_{*} \leq \frac{k(0)}{\mu}$ corresponds to a $T=\infty$ equilibrium. Therefore, by definition,

$$
\begin{aligned}
\frac{k(0)}{\mu} & \geq \frac{Q_{a-g}(\infty)}{\left[Q_{r-g}(\infty)\right]^{\frac{\alpha}{\alpha+\gamma}}} \\
& =Q_{a-g}(\infty) \frac{x(0)}{\mu} \text { by equation A; }
\end{aligned}
$$

so that rearrangement produces

$$
\frac{k(0)}{x(0)} \geq Q_{a-g}(\infty)=\frac{1}{a-g}
$$

which satisfies equation I. Thus, the conditions for equilibrium are satisfied.

By contrast, if $\psi_{*}>\frac{k(0)}{\mu}$, the final inequality above does not satisfy equation I.

\footnotetext{
${ }^{12}$ When $n \leq 0$ the limit is infinite.
} 
Lemma 5. For $\Psi(t)$ to be strictly quasiconcave, either of the following are sufficient:

1. $a \neq g$;

2. $\alpha>0$ and the Uzawa extraction condition holds.

Proof. Define

$$
D(t) \equiv \frac{d \ln \Psi(t)}{d t}=\frac{a-g}{e^{(a-g) t}-1}-\frac{\alpha}{\alpha+\gamma} \frac{r-g}{e^{(r-g) t}-1} .
$$

Therefore

$$
D^{\prime}(t)=-\frac{(a-g)^{2}}{\left[e^{(a-g) t}-1\right]^{2}}-\frac{\alpha}{\alpha+\gamma} \frac{(r-g)^{2}}{\left[e^{(r-g) t}-1\right]^{2}} .
$$

By l'Hôpital's rule, when $a=g$ or $r=g$, the whole term in which it is contained is zero. Thus, the stated conditions of the lemma suffice to ensure that either the first or second term of $D^{\prime}(t)$ is negative.

If either term is non-zero, the whole expression is strictly negative. This suffices for $\ln \Psi(t)$ to be strictly concave and, thus, for $\Psi(t)$ to be strictly quasiconcave.

Now prove the Theorem:

Proof. When $k(0) \in I_{L}, k(0) \geq 0$ and $\psi_{*}>\frac{k(0)}{\mu}$. Lemmata 1 and 2 and the continuity of $\Psi(t)$ ensure that there is a single finite $T$ such that $\Psi(T)=\frac{k(0)}{\mu}$. By Lemma 3, this implies a unique equilibrium with finite extinction date. As the inequality in $\psi_{*}$ is the reverse of the necessary and sufficient condition in Lemma 4, there are no equilibria with infinite extinction dates.

Now consider $k(0) \in I_{M} \Rightarrow \psi_{*}<\frac{k(0)}{\mu}<\psi^{*}$. The first of these ensures, by Lemma 4 , the existence of an equilibrium with an infinite extinction date. For the second inequality to hold, it must be that $\psi_{*}<\psi^{*}$. By the continuity of $\Psi(t)$ and the definition of $\psi^{*}$, there are two finite $T$ such that $\Psi(T)=$ $\frac{k(0)}{\mu}<\psi^{*}$. By Lemma 3, these are equilibria with finite extinction times.

When $k(0) \in I_{H}, \frac{k(0)}{\mu}>\psi^{*}$. Thus, by Lemma 3 , there are no equilibria with finite extinction times; by Lemma 4, there is one without extinction.

Now consider the degenerate cases. First, $k(0)=k_{L}=k_{H} \rightarrow \frac{k(0)}{\mu}=$ $\psi_{*}=\psi^{*}$. By Lemma 3, there is no equilibrium with finite extinction as $\psi_{*}$ is only reached as $T \rightarrow \infty$. Lemma 4 is satisfied with equality, producing an equilibrium without extinction.

Finally, when $k_{L} \neq k_{H}$, Lemma 4 is satisfied. Now a single finite $T$ satisfies Lemma 3, tangentially when $k(0)=k_{H}$. 


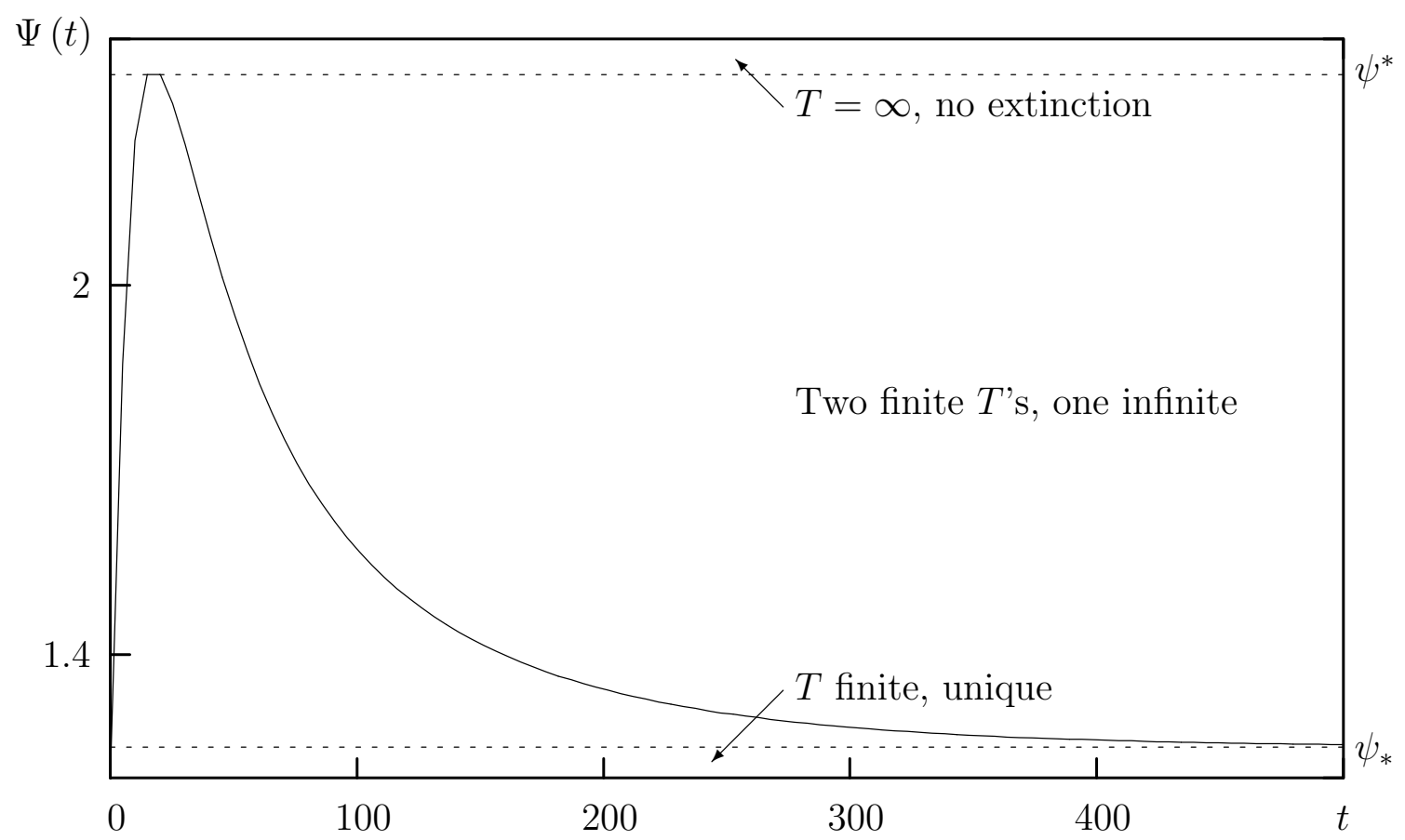

Figure 1: Extinction dates when $\alpha=\gamma=1, a=0.1, r=0.03$

Figure 1 shows an example. Multiplying the horizontal axis by $\mu$ allows $\Psi(t)$ to be replaced by $k(0)$, easing interpretation.

To this point, it has been assumed that $a>r$. While this presents the most interesting class of cases, its complement contains some canonical cases. Non-renewable resources, for which $a=0$ are the most obvious. These cases may be analysed using the objects already developed; in some cases, particular terms will be modified if their arguments are negative instead of positive.

Theorem 2. When the Uzawa extraction condition holds:

1. $I_{L}$ is always non-empty.

2. $I_{M}$ is non-empty iff $a>r$.

3. $I_{H}$ is empty when $a \leq g$.

Proof. 1. the continuity of $\Psi(t)$ and $\Psi(0)=0$ ensure the result if $\psi_{*}>0$. When $a>g$, this has already been demonstrated in Lemma 2. When 
$a=g$ and $r>g$

$$
\Psi(t)=t\left[\frac{r-g}{1-e^{-(r-g) t}}\right]^{\frac{\alpha}{\alpha+\gamma}} .
$$

Thus, the Uzawa extraction condition ensures that $\psi_{*}=\infty$. Finally, when $a<g, \lim _{t \rightarrow \infty} Q_{a-g}(t)=\infty$; as $\lim _{t \rightarrow \infty} Q_{r-g}(t), \psi_{*}$ is again infinite.

2. Sufficient conditions for the existence of $I_{M}$ are that $\Psi^{\prime}(t)=0$ for a finite $t$ and that $\Psi(t)$ be strictly quasiconcave. Consider all possible cases.

Under the Uzawa extraction condition, $a=g$ sets

$$
\Psi^{\prime}(t)=\frac{1}{\left[Q_{r-g}(t)\right]^{\frac{\alpha}{\alpha+\gamma}}}\left[1+\frac{\alpha}{\alpha+\gamma} \frac{t e^{-(r-g) t}}{Q_{r-g}(t)}\right]>0 \forall t>0 .
$$

Thus, $r>a=g$ suffices for an empty $I_{M}$.

Now consider $a \neq g$. Here

$$
\Psi^{\prime}(t)=\frac{e^{g t}}{Q_{r-g}(t)^{\frac{\alpha}{\alpha+\gamma}}}\left[e^{-a t}-\frac{\alpha}{\alpha+\gamma} \frac{Q_{a-g}(t)}{Q_{r-g}(t)} e^{-r t}\right] .
$$

Thus, a stationary point sets the square bracketed term to zero. Equivalently, it solves

$$
\frac{e^{r t}-e^{g t}}{e^{a t}-e^{g t}}=\frac{\alpha}{\alpha+\gamma} \frac{r-g}{a-g} .
$$

To simplify analysis, define

$$
\xi(t) \equiv \frac{e^{r t}-e^{g t}}{e^{a t}-e^{g t}}
$$

Thus, $\xi(t)$ is continuous for all $t \geq 0$ and, by l'Hôpital's rule, $\xi(0)=$ $\frac{r-g}{a-g}$. As this is greater in absolute value than the right hand side of equation 20 for all $\gamma>0$, a sufficient condition for an empty $I_{M}$ is that $(a-g) \xi^{\prime}(t) \geq 0 \forall t$.

Calculation yields

$$
\xi^{\prime}(t)=\frac{(r-a) e^{(a+r) t}-(r-g) e^{(r+g) t}+(a-g) e^{(a+g) t}}{\left(e^{a t}-e^{g t}\right)^{2}} .
$$

When $r>a>g$, this is positive. 
Now consider $r>g>a$. By Lemma $5, \Psi(t)$ is strictly quasiconcave. As $t \rightarrow \infty$, its denominator tends to $\left(\frac{1}{r-g}\right)^{\frac{\alpha}{\alpha+\gamma}}$, a positive finite number. Its numerator, however, tends to infinity. This, by strict quasiconcavity, precludes a maximum in finite $t$. Thus, $I_{M}$ is empty under these conditions.

Now consider $a=r>g$. In this case, the square bracketed term in equation 21 is identically zero, so that $\xi^{\prime}(t)=0 \forall t$. This suffices, from above, for an empty $I_{M}$.

Finally, consider $a>r>g$, the case considered above. In this case, the denominator of $\xi(t)$ grows more quickly than the numerator, so that $\xi(t)$ asymptotes to zero as $t \rightarrow \infty$.

3. from the first steps in the proof, $\psi_{*}=\infty$ when $a \leq g$. With the Uzawa extraction condition, this suffices for an infinite $\psi^{*}$.

The non-extinction equilibrium may be explicitly eliminated by noting that allowing for $a<g$ replaces equation I with

$$
Q_{a-g}(T)=\min \left\{\frac{k(0)}{x(0)}, \max \left\{\frac{1}{a-g}, 0\right\}\right\} .
$$

Thus, the final inequality in Lemma 4 requires $x(0)=0$. Equation $\mathrm{A}$, in turn, then requires $\mu=0$, a contradiction by definition of $\mu$ and $\nu$.

Now consider the limit case in which $\gamma \rightarrow 0$. This seems to correspond to that studied in Gaudet et al. (2002). In this case, extraction costs reflect only total extraction, rather than its rate. Thus, pulse extraction is no more costly than smooth extraction. Thus, "the extraction contest is so fierce that the common is drained in the instant storage is initiated" (Gaudet et al., 2002).

Theorem 3 (Gaudet et al. (2002) 'jump extinction'). The extinction date, $T$, goes to zero with $\gamma$.

The first condition includes $a=r$, the costless storage of Gaudet et al. (2002).

Proof. Assume that $T$ is finite. Then, under the stated conditions, equations A and I combine to yield

$$
\Psi(T)=\frac{k(0)}{\mu}=\frac{Q_{a-g}(T)}{Q_{r-g}(T)^{\frac{\alpha}{\alpha+\gamma}}} .
$$


This may be rearranged and rewritten in terms of primitives for

$$
\frac{k(0)}{\mu} \frac{r+a \gamma-\rho}{[(1+\gamma) r-\rho]^{\frac{\alpha}{\alpha+\gamma}}} \gamma^{-\frac{\gamma}{\alpha+\gamma}}=\left[1-e^{-\frac{r+a \gamma-\rho}{\gamma} T}\right]\left[1-e^{-\frac{(1+\gamma) r-\rho}{\gamma} T}\right]^{-\frac{\alpha}{\alpha+\gamma}}
$$

so that, as $\lim _{\gamma \rightarrow 0} \gamma^{-\frac{\gamma}{\alpha+\gamma}}$,

$$
\frac{k(0)}{\mu}=\lim _{\gamma \rightarrow 0}\left[1-e^{-\frac{r+a \gamma-\rho}{\gamma} T}\right]\left[1-e^{-\frac{(1+\gamma) r-\rho}{\gamma} T}\right]^{-\frac{\alpha}{\alpha+\gamma}} .
$$

If $T$ remained positive as $\gamma \rightarrow 0$, then the right hand side of the equation would converge to unity, a contradiction. Thus, $T \rightarrow 0$ as $\gamma$ does.

Thus, jump extinctions only require that extraction costs become linear. Unlike Gaudet et al. (2002), there is no condition on the cost of storage. The difference between these results does not seem reflect the difference between storage alone and full capital market access: agents are not taking advantage of their ability to borrow against future income here. Instead, they are banking and saving it all initially.

\section{The commons without capital markets}

This section compares the RE equilibria with capital market access to those without such access.

When individuals do not have access to capital markets, their consumption and effort smoothing problems are addressed as a unit. Intertemporal budget constraint 3 is replaced by $c_{i}(t)=x_{i}(t)$; feasibility constraint 4 remains the same. Thus, agents maximise

$$
\int_{0}^{\infty} e^{-\rho t}\left[\frac{x_{i}(t)^{1-\alpha}}{1-\alpha}-\frac{1}{\theta_{i}} \frac{x_{i}(t)^{1+\gamma}}{1+\gamma}\right] d t \text { subject to constraint } 4 .
$$

Maximising produces

$$
x_{i}(t)=\left\{\begin{array}{ll}
\theta_{i}^{\frac{1}{\alpha+\gamma}} & \text { for } \quad t<T \\
0 & \text { for } \quad t \geq T
\end{array}\right\} .
$$

Integrating over all agents yields aggregate extraction

$$
\tilde{x}(t)=\left\{\begin{array}{ll}
\mu \nu^{-\frac{1}{\alpha+\gamma}} & \text { for } \quad t<T \\
0 & \text { for } \quad t \geq T
\end{array}\right\}
$$


The tilde distinguishes this solution from that with capital market access. As agents are unable to intertemporally smooth, they maximise myopically, extracting at the instantaneously optimal rate without consideration of the stock consequences. Thus, extraction is also independent of the initial capital stock.

With equation of motion 2 , direct solution for the extinction date allows

$$
\tilde{T}=\frac{1}{a}\left\{\ln [\mu]-\ln \left[\mu-a \nu^{\frac{1}{\alpha+\gamma}} k(0)\right]\right\} .
$$

Again, it is assumed that the Uzawa conditions derived earlier hold: the term in $\nu$ may not be real valued otherwise.

Thus, $\tilde{T}$ increases in $k(0)$, approaching infinity asymptotically as $k(0) \nearrow$ $\frac{\mu}{a \nu^{\frac{1}{\alpha+\gamma}}}$. Stocks of $k(0)$ above this level are never depleted. Further, $\tilde{T}$ is convex in $k(0)$.

Lemma 6. A necessary condition for $\tilde{x}(0)>x(0)$ is

$$
\frac{\gamma}{(1+\gamma) r-\rho}>\left[\frac{\alpha}{\rho-(1-\alpha) r}\right]^{\alpha}
$$

There are always values of $k(0)$ such that $\tilde{x}(0)<x(0)$.

Proof. By equations A and 23, $\tilde{x}(0)>x(0)$ requires

$$
\left[Q_{r-g}(T)\right]^{\alpha}>\nu
$$

As the right hand side is constant in $k(0)$, consider the left hand side. Its derivative in $T$ is positive, so that a necessary condition for the inequality to hold is that it hold at

$$
k(0)=\infty \Rightarrow T=\infty \Rightarrow Q_{r-g}(\infty)=\frac{1}{r-g} .
$$

The condition follows from the definitions of $g$ and $\nu$.

The second part of the lemma follows from noting that

$$
k(0)=0 \Rightarrow T=0 \rightarrow Q_{r-g}(0)=0<\nu
$$

when the Uzawa consumption condition holds.

Figure 2 displays an example of the effect of capital market access on extinction dates. ${ }^{13}$ The curve referring to capital market access is that in Figure

\footnotetext{
${ }^{13}$ Maple code available from the authors upon request.
} 


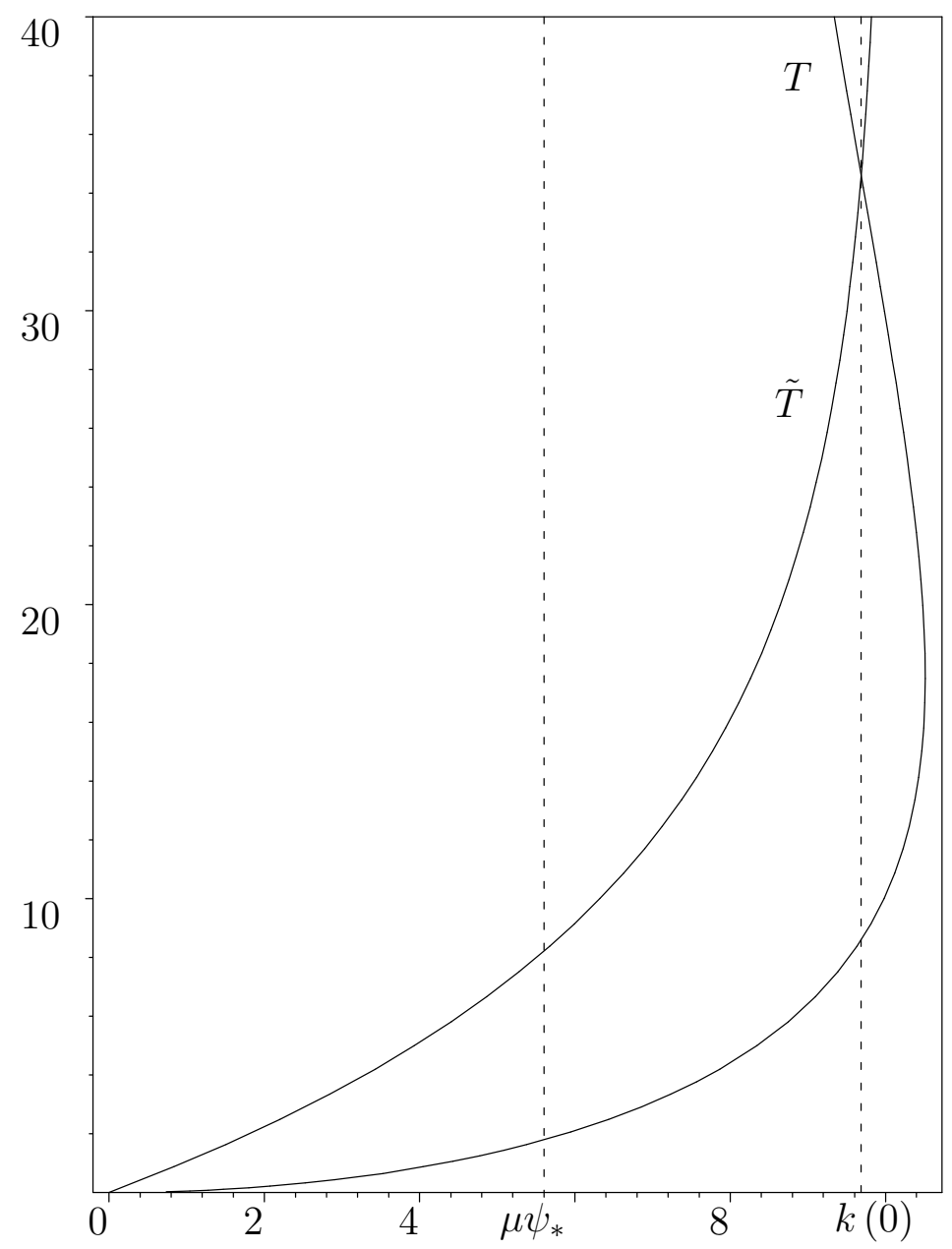

Figure 2: Extinction dates varying in $k(0)$ when $\alpha=\gamma=1, \rho=0.05, \theta_{i}=$ $1 \forall i, a=0.1, r=0.03$

1. Here, low levels of commons stock are preserved for longer by individuals without access to capital markets. Above $k(0)=\mu \psi_{*}$, an intermediate zone is entered. In this, the extinction date without capital market access lies between the two finite extinction dates with capital market access. At the same time, there is an equilibrium with no extinction when individuals have access to capital markets.

Finally, above a higher level of $k(0)$, the extinction date without capital market access is greater than both of the finite dates with access. Again, though, there is a non-extinction equilibrium with capital market access.

Extinction dates are poor proxies for welfare: late extinction dates are obtained by low extraction rates. Consider a situation in which the commons 
is exhausted one period earlier under capital market access than it is without it. In this case, one period of returns at rate $a$ are lost, but the privately stored extraction in that period then grows at rate $r$. By contrast, in the situation without capital market access, the extra period of $a$ growth is not balanced by future $r$ growth. Thus, we now compare welfare directly.

The equilibrium welfare obtained by the infinitesimal agents $d i$ with access to capital markets may be expressed in terms of initial extraction, $x_{i}(0)$ by substitution with equations 8,11 and 12 :

$$
W_{i}=\theta_{i}^{\frac{1-\alpha}{\alpha+\gamma}} \nu^{\frac{1+\gamma}{\alpha+\gamma}}\left[Q_{r-g}(T)\right]^{\frac{1-\alpha}{\alpha+\gamma} \gamma} \frac{\alpha+\gamma}{(1-\alpha)(1+\gamma)} .
$$

As $T$ is not generally a function of $k(0)$, this cannot be expressed as a function of $k(0)$ directly.

The welfare obtained by individuals $d i$ without access to capital markets may also be calculated:

$$
\begin{aligned}
\tilde{W}_{i} & =\frac{1}{\rho} \frac{\alpha+\gamma}{(1-\alpha)(1+\gamma)} \theta_{i}^{\frac{1-\alpha}{\alpha+\gamma}}\left[1-e^{-\rho \tilde{T}}\right] \\
& =\frac{1}{\rho} \frac{\alpha+\gamma}{(1-\alpha)(1+\gamma)} \theta_{i}^{\frac{1-\alpha}{\alpha+\gamma}}\left[1-\left(\frac{\mu}{\mu-a \nu^{\frac{1}{\alpha+\gamma}} k(0)}\right) e^{-\frac{\rho}{a}}\right] .
\end{aligned}
$$

Figure 3 displays the results for the same parameter values as those used above. The horizontal line extending across the whole domain corresponds to welfare in the standard autarkic equilibrium. This this has a nice interpretation - all rents are dissipated regardless of initial commons stock - it here reflects $\alpha=1$ and $\theta_{i}=1$.

The rising curve, becoming a horizontal line, represents welfare with access to capital markets. The section of the curve that decreases in $k(0)$ may be thought of as a resource curse segment: here, the effect is driven by the expectation that the 'boon' of additional $k(0)$ will cause others to increase their extraction of it sufficiently to reduce its extinction date. This, in turn, causes individuals to increase their own extraction rates. This 'curse', however, still yields higher welfare than does the upward sloping segment.

Comparing the levels of welfare under autarky and with capital market access, it may be seen that the autarkic equilibrium dominates for all $k(0)<$ $\mu \psi_{*}$. Below that point, agents with capital market access expect such a rapid extinction that they undertake very rapid, thus expensive, extraction themselves, banking the proceeds.

Beyond that point, there are equilibria with capital market access that dominate the autarkic. At the point at which the equilibrium with most rapid extinction comes to dominate the autarkic, its extinction date is still much shorter than the autarkic's, justifying our earlier conjecture. 


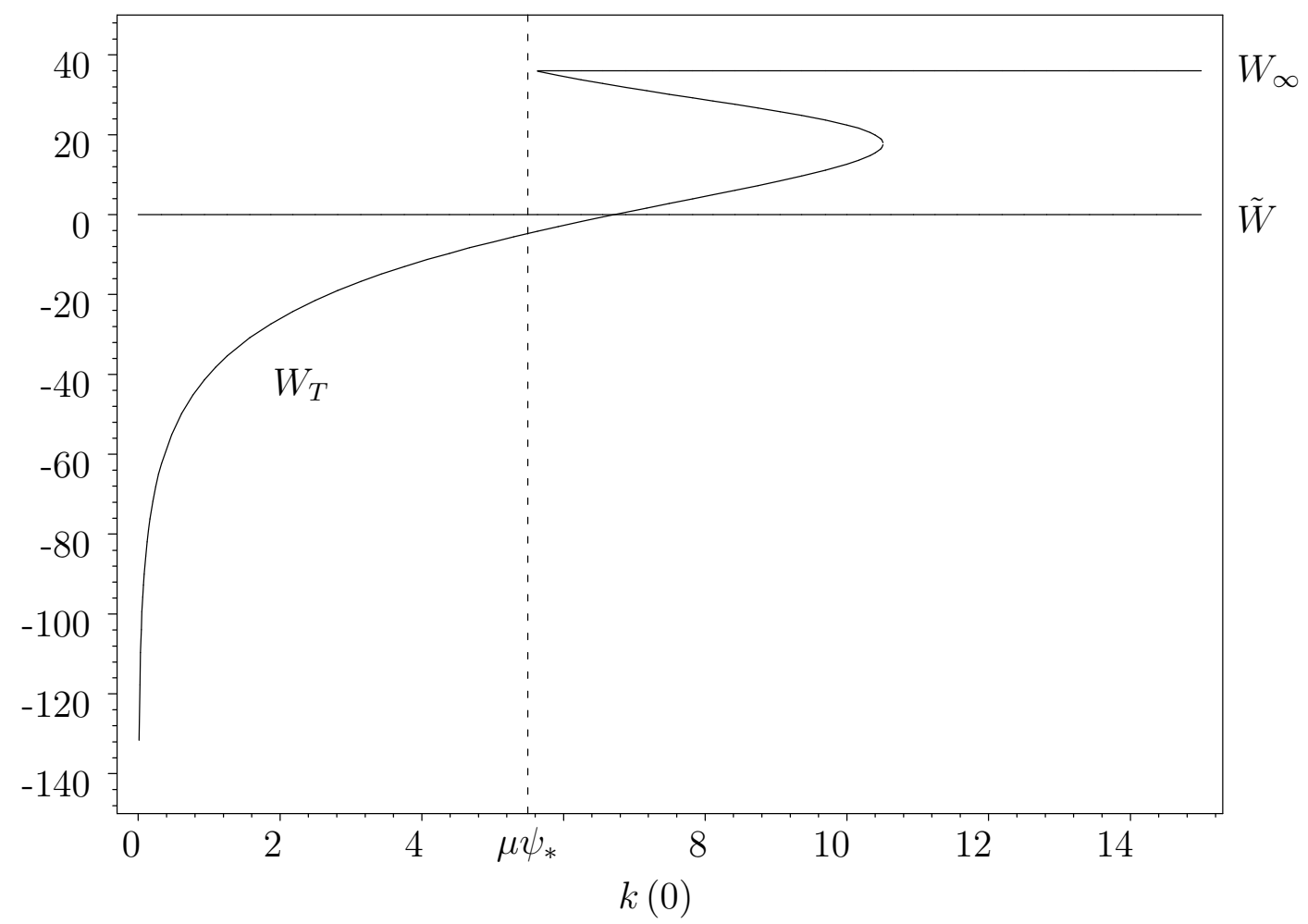

Figure 3: Welfare varying in $k(0)$ when $\alpha=\gamma=1, \rho=0.05, \theta_{i}=1 \forall i, a=$ $0.1, r=0.03$

\section{Taxation}

Suppose now that the government imposes an extraction tax, $\delta$, so that infinitesimal agents $d i$ retain $x_{i}^{\delta}=(1-\delta) x_{i}$ after having extracted quantity $x_{i}$.

In the case of literal commons, tax revenue earned might be spent providing public goods. We, however, follow the Tornell and Velasco (1992) interpretation, and consider institutional commons. Tax revenue, $\delta x$, is returned to the commons. Thus, taxes both reduce agents' productivity from $\theta_{i}$ and replenish the commons.

The equations in Section 3 may largely be rewritten in terms of $x_{i}^{\delta}$ instead of $x_{i}$. The consumption smoothing problem is subject to the feasibility constraint

$$
X_{i}^{\delta}(r)=\int_{0}^{\infty} e^{-r t} x_{i}^{\delta}(t) d t
$$

which replaces $X_{i}$ in subsequent calculations. The consumption calculations are otherwise unchanged. 
As to extraction, first order condition 12 becomes

$$
x_{i}^{\delta}(0)^{\alpha+\gamma}=\frac{\theta_{i} \nu}{\left[Q_{r-g}\left(T^{\delta}\right)\right]^{\alpha}}(1-\delta)^{1-\alpha} ;
$$

while the first fundamental equation, $\mathrm{A}$, is now

$$
x^{\delta}(0)=\frac{\mu}{\left[Q_{r-g}\left(T^{\delta}\right)\right]^{\frac{\alpha}{\alpha+\gamma}}}(1-\delta)^{\frac{1-\alpha}{\alpha+\gamma}} .
$$

The extraction rate relative to the situation without a tax therefore depends on two factors: a direct effect, and an indirect effect through the extinction date.

The second fundamental equation, I, becomes

$$
Q_{a-g}\left(T^{\delta}\right)=\min \left\{\frac{1}{1-\delta} \frac{k(0)}{x(0)}, \frac{1}{a-g}\right\}
$$

Thus, for finite $T^{\delta}$, the effect of an extraction tax is to 'inflate' $k(0)$. Whether this increases or decreases the extinction date depends on the equilibrium selected.

Theorem 1 goes through when $k_{L}$ and $k_{H}$ are replaced by $k_{L}^{\delta} \equiv \mu \psi_{*}(1-\delta)^{\frac{1+\gamma}{\alpha+\gamma}}$ and $k_{H}^{\delta} \equiv \mu \psi^{*}(1-\delta)^{\frac{1+\gamma}{\alpha+\gamma}}$. This, in turn, alters the conditions in Lemmata 3 and 4 . Otherwise, the remaining results go through unchanged.

As $(1-\delta)^{\frac{1+\gamma}{\alpha+\gamma}}<1$ for all $\delta>0$, the effect of an extraction tax is to reduce toward zero the boundaries of the intervals defined in Theorem 1. Some initial capital stocks that, without taxation, were in $I_{L}\left(\right.$ resp. $\left.I_{M}\right)$ are, with taxation, in $I_{M}\left(r e s p . I_{H}\right)$. Thus, taxation increases the set of $k(0)$ over which non-extinction is possible.

The welfare calculations in equation 25 may be altered for

$$
\begin{aligned}
W_{i}^{\delta}=\theta_{i}^{\frac{1-\alpha}{\alpha+\gamma}} \nu^{\frac{1+\gamma}{\alpha+\gamma}} & \left\{\frac{1}{1-\alpha}(1-\delta)^{\frac{(1-\alpha)^{2}}{\alpha+\gamma}}\left[Q_{r-g}\left(T^{\delta}\right)\right]^{-\frac{1-\alpha}{\alpha+\gamma} \alpha}\right. \\
& \left.-\frac{1}{1+\gamma}(1-\delta)^{\frac{(1-\alpha)(1+\gamma)}{\alpha+\gamma}}\left[Q_{r-g}\left(T^{\delta}\right)\right]^{\frac{1-\alpha}{\alpha+\gamma} \gamma}\right\} .
\end{aligned}
$$

The effect of taxation appears in both the consumption and extraction terms.

Figure 4 displays the welfare consequences of an extraction tax. This is set at $\delta=.5$ for illustrative purposes; the other parameters are as they were in earlier figures.

Without extinction, welfare is not altered by the tax in this example. This is a consequence of $\alpha=1$ : when this holds, the only difference between 


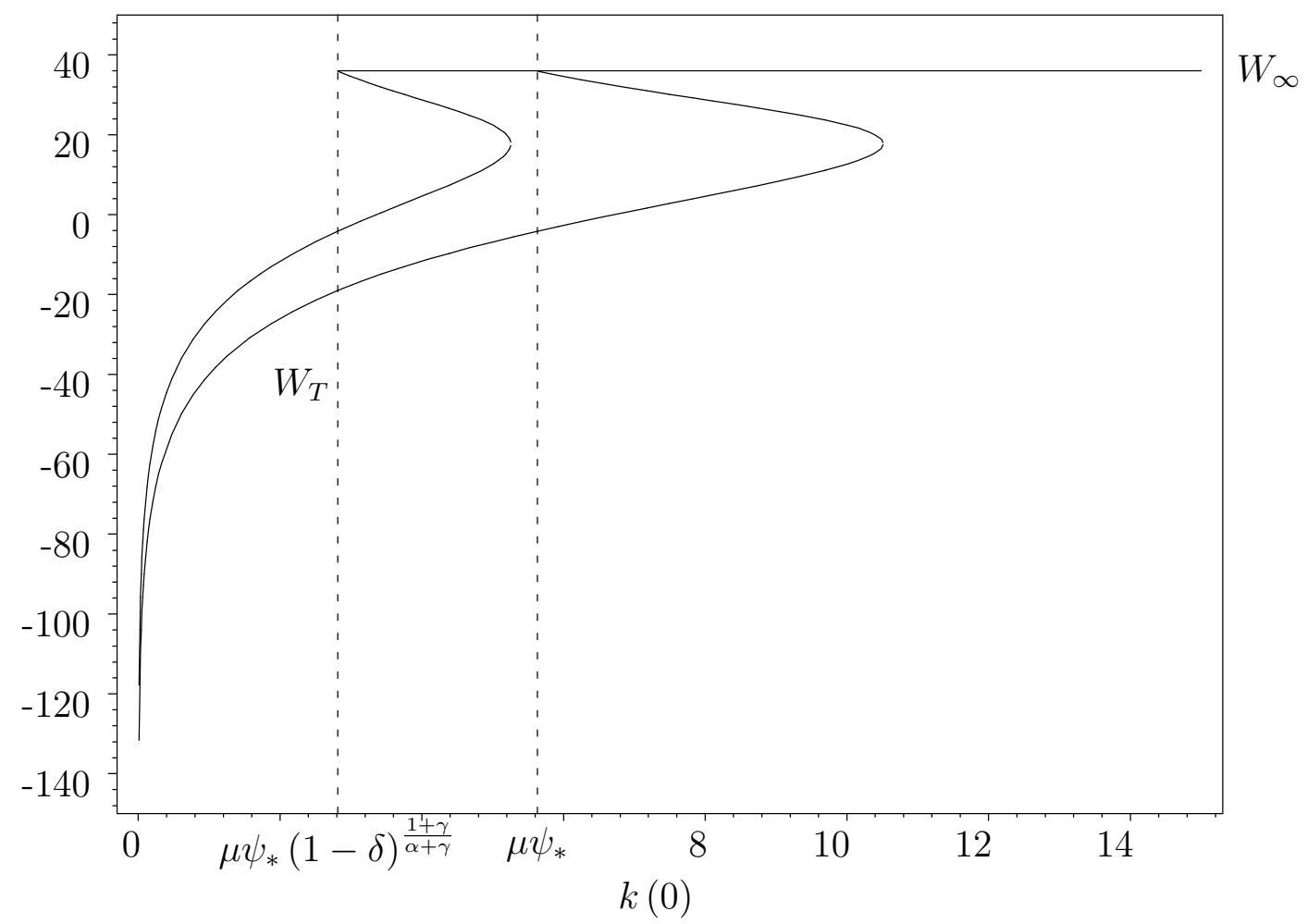

Figure 4: Welfare consequences of $\delta=.5$; other parameters as before

equation 26 and equation 25 owes to differing extinction dates. Without extinction, this example leaves extraction rates unchanged.

The finite extinction equilibrium in which welfare increases in $k(0)$ dominates that without an extraction tax. In this case, the replenishment effect of the extraction tax seems to dominate its reduced productivity effect.

Finally, in the cursed equilibrium, taxation worsens welfare. This effect is mediated by hastened extinction. More positively, the domain over which the cursed equilibrium is possible is reduced by taxation. In the extreme, at $\delta=1$, the $I_{M}$ interval disappears, eliminating the cursed equilibrium.

A consumption tax may also be considered using similar techniques. Supposing that the tax that reduces consumption to $c_{i}^{\varepsilon}=(1-\varepsilon) c_{i}$ modifies equation 1 for

$$
\dot{k}(t)=a k(t)-x(t)+\varepsilon c(t), k(0)>0 .
$$

All the steps taken above may be repeated. As the equation of motion is now more complicated, so is the new version of equation I.

Savings taxes are more difficult to consider. These would reduce $x_{i}-c_{i}$ when this difference was positive, but not otherwise, introducing a kink into agents' problems. Such a tax corresponds most closely to capital controls. 


\section{Discussion}

We have analysed extraction from a commons when agents have access to capital markets. Comparison to the standard in the literature, in which agents do not have such access, shows that the results can differ significantly. Qualitatively, the difference appears to be largest when the resource in the commons grows quickly. A further comparison that may be of interest, given the existing literature, would be to the situation with storage.

The extraction tax considered above may be thought of as decreasing individuals' $\theta_{i}$, increasing their extraction costs. At the outset of the taxation discussion, it was noted that a literal understanding of the commons might not return tax revenue raised to the commons. Under this interpretation, governments may spend it on public goods of various sorts. Within the framework of this model, the one parameter that might be influenced by such expenditures is individuals' productivity, $\theta_{i}$. If tax revenue increased $\theta_{i}$, then the welfare consequences of the tax might be the opposite of those developed above.

Three generalisations seem fairly natural. First, the specification of the constant $a$ is, in general, an over-simplification. Biological models typically allow the growth rates to be functions of the stock (q.v. Dockner and Sorger, 1996; Kremer and Morcom, 2000), $a(k)$ in this case. Implications of this generalisation are sketched here.

The equations underlying equation $\mathrm{A}$ are unchanged. Equation 2, however, becomes

$$
k(t)=\max \left\{0, e^{\int_{0}^{t} a(k(\tau)) d \tau}\left[k(0)-\int_{0}^{t} e^{-\int_{0}^{\tau} a(k(\sigma)) d \sigma} x(\tau) d \tau\right]\right\} .
$$

Thus, equation 14 becomes

$$
k(0)=x(0) \int_{0}^{T} e^{g t-\int_{0}^{t} a(k(\tau)) d \tau} d t .
$$

Integrability requires a new Uzawa extraction condition; the equivalent of equation I will no longer be a clear expression of $Q_{a-g}(T)$.

A second generalisation involves analysis of strategic agents, as in Tornell and Velasco (1992) and Tornell and Lane (1999). Strategic analysis requires calculating optimal strategies for every combination of private savings and commons stock that can be reached from the problem's initial conditions. Thus, the Hamilton-Jacobi-Bellman equation is a PDE in private savings and commons stock.

A final generalisation involves considering the possibility of default by borrowing agents. 


\section{References}

Jess Benhabib and Roy Radner. The joint exploitation of a productive asset: a game-theoretic approach. Economic Theory, 2(2):155 - 190, 1992.

Hernando de Soto. The Mystery of Capital. Bantam Press, 2000.

E. Dockner, S. Jørgenson, N. V. Long, and G. Sorger. Differential games in economics and management science. Cambridge University Press, 2000.

E. J. Dockner and G. Sorger. Existence and properties of equilibria for a dynamic game on productive assets. Journal of Economic Theory, 71: 209-27, 1996.

Jayasri Dutta and Colin Rowat. Pillaging the commons: weak property rights and private savings. mimeo, 9 December 2003.

P. K. Dutta and R. K. Sundaram. The tragedy of the commons? Economic Theory, 3(3):413-26, 1993.

D. Fudenberg and J. Tirole. Capital as commitment: strategic investment to deter mobility. Journal of Economic Theory, 31:227 - 256, 1983.

Gérard Gaudet, Michel Moreaux, and Stephen W. Salant. Private storage of common property. Journal of Environmental Economics and Management, $43: 280-302,2002$.

H. Scott Gordon. The economic theory of a common-property resource: The fishery. Journal of Political Economy, 62(2):124 - 142, April 1954.

Frances R. Homans and James E. Wilen. Markets and rent dissipation in regulated open access fisheries. mimeo, March 2001.

Harold Houba, Koos Sneek, and Felix Várdy. Can negotiations prevent fish wars? Journal of Economic Dynamics and Control, 24:1265 - 1280, 2000.

Michael Kremer and Charles Morcom. Elephants. American Economic Review, 90(1):212 - 234, March 2000.

D. Levhari and L. J. Mirman. The great fish war: an example using a dynamic Cournot-Nash solution. Bell Journal of Economics, 11:322-334, 1980.

Leonard J. Mirman. Dynamic Models of Fishing: A heuristic approach, pages 39-73. Dekker, 1979. 
Paul Richter. Contractors' risks, costs high in Iraq. Los Angeles Times, 25 December 2003.

Michael Ross. Natural Resources and Violent Conflict: Options and Actions, chapter The Natural Resource Curse: How Wealth Can Make You Poor, pages 17 - 42. The World Bank, 2003.

Xavier Sala-i-Martin and Arvind Subramanian. Addressing the natural resource curse: an illustration from Nigeria. Working Paper 9804, NBER, June 2003.

H.-W. Sinn. Common property resources, storage facilities and ownership structures: a cournot model of the oil market. Economica, 51:235 - 252, August 1984.

G. Sorger. Markov-perfect nash equilibria in a class of resource games. Economic Theory, 11(1):79-100, 1998.

Aarón Tornell and Philip R. Lane. The voracity effect. American Economic Review, 89(1):22 - 46, March 1999.

Aarón Tornell and Andrés Velasco. The tragedy of the commons and economic growth: why does capital flow from poor to rich countries? Journal of Political Economy, 100(6):1208 - 1231, 1992.

Wolfgang Walter. Ordinary Differential Equations, volume 182 of Graduate Texts in Mathematics. Springer, 1998.

Kenneth R. Weiss. Fish farms become feedlots of the sea. Los Angeles Times, 9 December 2002. 